\title{
Emittance fluctuations in a mesoscopic diffusive conductor
}

\author{
Tiago De Jesus, ${ }^{1}$ Hong Guo, ${ }^{1}$ and Jian Wang ${ }^{2}$ \\ ${ }^{1}$ Center for the Physics of Materials and Department of Physics, McGill University, Montreal, PQ, Canada H3A 2T8 \\ ${ }^{2}$ Department of Physics, The University of Hong Kong, Pokfulam Road, Hong Kong, China
}

(Received 28 April 2000)

\begin{abstract}
We report a first principles analysis of low frequency dynamic conductance fluctuations for disordered two-dimensional mesoscopic conductors. In the transport regime where dc conductance shows the familiar universal conductance fluctuations, we discovered that the low frequency emittance also fluctuates with an amplitude that is independent of the impurity scattering strength, showing a degree of generic behavior. When the impurity density is increased such that the dynamic response of the conductor changes from inductivelike to capacitivelike, the emittance distribution is found to cross over from Gaussian-like to non-Gaussian-like; the latter is qualitatively consistent with random matrix theory.
\end{abstract}

One of the most striking phenomena of transport in mesoscopic regime is the observed universal conductance fluctuations ${ }^{1}$ (UCF's). These fluctuations are not time dependent noise but are reproducible signatures of quantum interference. The physics of the sample-to-sample dc conductance fluctuations and their universal behavior have been the subject of active research for more than a decade ${ }^{2}$ and is now well understood. From a larger physical point of view, the dc conductance of a conductor is the zero frequency limit of the ac admittance $G_{\alpha \beta}(\omega)$, where $\alpha$ and $\beta$ label the leads of the conductor. Since for a diffusive conductor $G_{\alpha \beta}(0)$ shows UCF's in the mesoscopic regime, it is very interesting to ask: What are the sample-to-sample statistical properties of the ac admittance?

For a multiterminal mesoscopic conductor, theoretical analysis of its linear dc conductance is considerably simplified due to the fact that $G_{\alpha \beta}(0)$ depends only on the equilibrium electrostatic potential but not on the potential that is established in the presence of transport inside the conductor. Analysis of the ac admittance $G_{\alpha \beta}(\omega)$ has proven to be quite nontrivial $^{3,4}$ as it is a functional of the potential buildup inside the conductor. Because of this dependence, one expects the sample-to-sample statistical properties of $G_{\alpha \beta}(\omega)$ to be less universal than those of $G_{\alpha \beta}(0)$. Experimental studies of dynamic conductance fluctuations in mesoscopic rings have been reported by Pieper and Price. ${ }^{5}$ Theoretical investigations have been carried out on a number of topics related to fluctuations of dynamic conductance, including the fluctuating admittance of chaotic cavities, ${ }^{6,7}$ random matrix analysis of capacitance distribution, ${ }^{8}$ transfer matrix studies of low frequency quasi-one-dimensional systems, ${ }^{9}$ and dynamic magnetoconductance fluctuations. ${ }^{10}$

In this paper, we present a theoretical investigation of sample-to-sample statistical properties of the low frequency admittance. Our analysis is based on the theory of Büttiker, Prêtre, and Thomas, ${ }^{3}$ and our calculation is from first principles by evaluating the internal potential response from the density of states $^{11}$ (see below), rather than using the approximate constant capacitance charging model. In particular we focus on the quantity called the emittance, $E_{\alpha \beta}$, defined ${ }^{11}$ by the low frequency expansion of the admittance,

$$
G_{\alpha \beta}(\omega)=G_{\alpha \beta}(0)-i \omega E_{\alpha \beta}+O\left(\omega^{2}\right) .
$$

$E_{\alpha \beta}$ measures the dynamic response of the system to an external time dependent perturbation: the response is capacitive if $E_{11}$ is positive while it is inductive when $E_{11}$ is negative. For a conductor capacitively coupled to an external gate, $E_{11}$ is just the electrochemical capacitance. ${ }^{11}$ For a onedimensional (1D) diffusive conductor, the average value of $E_{11}$ is discussed in a recent review of Büttiker and Christen ${ }^{12}$ and was found to be zero by solving the classical diffusion equation for which the weak localization effect was neglected. When the weak localization effect is included, as predicted in Ref. 6 for chaotic cavities, the average of $E_{11}$ is nonzero.

For a disordered conductor described by the elastic mean free path $l$ and conductor linear size $L$, the quantity $l / L$ has been considered ${ }^{13}$ as the fraction of all the $M$ transmission channels in the disordered sample (e.g., in 2D) for which the transmission probability is of order unity, i.e., these $M_{\text {eff }}$ $\sim M(l / L)$ channels are the open channels responsible for conduction. The conventional UCF phenomenon can be viewed as a reflection of the sample-to-sample fluctuations of the number ${ }^{13} M_{e f f}$. For highly disordered samples $l \ll L$; they have large resistance and therefore we expect a capacitivelike dynamic response. On the other hand, when the degree of disorder is reduced the response can be inductivelike. Hence we expect the distribution function for $E_{11}$, which will be calculated below, to change as the degree of disorder changes, indicating a crossover from that reflecting a capacitive response to that of an inductive response.

To be specific we consider single electron transport through a 2D conductor whose disorder is provided by an impurity scattering potential $V_{I}(\mathbf{r})=\Sigma_{i} \gamma_{i} \delta\left(\mathbf{r}-\mathbf{r}_{i}\right)$, where $\gamma_{i}$ is the strength of the $i$ th impurity located at position $\mathbf{r}_{i}$. For simplicity we fix $\gamma_{i}=\gamma$ as an input parameter of the analysis. The emittance of a 2D conductor with a single impurity has been calculated exactly before using scattering matrix theory, ${ }^{14,15}$ but a direct extension of this approach to an $N$-impurity problem is very difficult. We hence developed a Green's function technique to solve this problem. The low frequency emittance can be written as ${ }^{16} E_{\alpha \beta}=d N_{\alpha \beta} / d E$ $-D_{\alpha \beta}$, where the term $d N_{\alpha \beta} / d E$ is the global partial den- 
sity of states (GPDOS) ${ }^{17}$ The term $D_{\alpha \beta}$ is due to the Coulomb interaction of electrons inside the sample, and it can be computed from the local density of states. ${ }^{16}$ With the Thomas-Fermi approximation one can prove ${ }^{16}$

$$
D_{\alpha, \beta}=\int d^{3} r \frac{[d n(\alpha, \mathbf{r}) / d E][d n(\mathbf{r}, \beta) / d E]}{d n(\mathbf{r}) / d E},
$$

where the local density of states $d n(\alpha, \mathbf{r}) / d E$ $=d n(\mathbf{r}, \beta) / d E$ in zero magnetic field ${ }^{17}$ and $^{16}$

$$
\frac{d n(\alpha, \mathbf{r})}{d E}=\sum_{\beta} \frac{d n_{\alpha \beta}(\mathbf{r})}{d E},
$$

and the local partial DOS (LPDOS) is given by

$$
\frac{d n_{\alpha \beta}(\mathbf{r})}{d E}=-\frac{1}{4 \pi i} \operatorname{Tr}\left(\mathbf{s}_{\alpha \beta}^{\dagger} \frac{\delta \mathbf{s}_{\alpha \beta}}{\delta U(\mathbf{r})}-\frac{\delta \mathbf{s}_{\alpha \beta}^{\dagger}}{\delta U(\mathbf{r})} \mathbf{s}_{\alpha \beta}\right) .
$$

Finally, the GPDOS is calculated from the LPDOS by appropriate spatial integration over the conductor,

$$
\frac{d N_{\alpha \beta}}{d E}=\int d \mathbf{r} \frac{d n_{\alpha \beta}(\mathbf{r})}{d E} .
$$

From the theoretical formalism outlined above, a first principles analysis of emittance necessarily requires the calculation of functional derivatives of the scattering matrix with respect to a variation of the scattering potential landscape, e.g., Eq. (4). In this work this is achieved by a Green's function formalism. ${ }^{19}$ Briefly, for a $2 \mathrm{D}$ conductor with the $N$-impurity scattering potential $V_{I}(\mathbf{r})$, we first calculate its Green's function by iterating the Dyson equation

$$
G^{(N)}\left(\mathbf{r}, \mathbf{r}^{\prime}\right)=G^{(0)}\left(\mathbf{r}, \mathbf{r}^{\prime}\right)+\gamma \sum_{i=1}^{N} G^{(0)}\left(\mathbf{r}, \mathbf{r}_{i}\right) G^{(N)}\left(\mathbf{r}_{i}, \mathbf{r}^{\prime}\right),
$$

where $G^{(N)}\left(\mathbf{r}, \mathbf{r}^{\prime}\right)$ and $G^{(0)}\left(\mathbf{r}, \mathbf{r}^{\prime}\right)$ are the $N$-impurity and zero-impurity Green's functions, respectively. $G^{(0)}\left(\mathbf{r}, \mathbf{r}^{\prime}\right)$ is simply the Green's function for an infinitely long quasi-1D ballistic quantum wire (along the $x$ direction), ${ }^{18}$

$$
G^{(0)}\left(\mathbf{r}, \mathbf{r}^{\prime}\right)=\sum_{n=1} \phi_{n}(y) \phi_{n}\left(y^{\prime}\right) \frac{e^{i k_{n}\left|x-x^{\prime}\right|}}{2 i k_{n}},
$$

where $\phi_{n}(y)$ is the $n$th mode of the transverse wave function and $k_{n}$ the corresponding momentum of the electron. The Dyson equation can be solved exactly and we obtain

$$
G^{(N)}\left(\mathbf{r}, \mathbf{r}^{\prime}\right)=G^{(0)}\left(\mathbf{r}, \mathbf{r}^{\prime}\right)+\gamma \sum_{i, j=1}^{N} G^{(0)}\left(\mathbf{r}, \mathbf{r}_{i}\right) M_{i j} G^{(0)}\left(\mathbf{r}_{j}, \mathbf{r}^{\prime}\right),
$$

where the matrix $M_{i j} \equiv\left[\delta_{i j}-\gamma \eta_{i j}\right]^{-1}$ and $\eta_{i j} \equiv G^{(0)}\left(\mathbf{r}_{i}, \mathbf{r}_{j}\right)$. With the Green's function calculated this way we obtain the scattering wave function via the Lippmann-Schwinger equation. The result is

$$
\psi^{(N)}(\mathbf{r})=\psi^{(0)}(\mathbf{r})+\gamma \sum_{i, j=1}^{N} G^{(0)}\left(\mathbf{r}, \mathbf{r}_{i}\right) M_{i j} \psi^{(0)}\left(\mathbf{r}_{j}\right),
$$

where $\psi^{(N)}(\mathbf{r})$ and $\psi^{(0)}(\mathbf{r})$ are the $N$-impurity and zeroimpurity wave functions, respectively. Moreover, the functional derivative of the scattering wave function can be calculated ${ }^{19}$ by investigating its linear response to an infinitesimal perturbation $\delta U(\mathbf{r})=\delta U \delta\left(\mathbf{r}-\mathbf{r}^{\prime}\right)$, where $\delta U \rightarrow 0$. We apply the Lippmann-Schwinger equation to obtain an expression for the full wave function response to this perturbation, and then expand this expression in terms of $\delta U$. The first order term gives the functional derivative of the wave function, which is found to be

$$
\frac{\delta \psi^{(N)}\left(\mathbf{r}^{\prime}\right)}{\delta U(\mathbf{r})}=G^{(N)}\left(\mathbf{r}^{\prime}, \mathbf{r}\right) \psi^{(N)}(\mathbf{r}) .
$$

The scattering matrix and its functional derivatives can now be extracted from the scattering wave function. In closed form we obtain

$$
\begin{gathered}
\mathbf{s}_{1 n, 1 m}=\sum_{j, l=1}^{N} \beta_{j l}^{n m m} \frac{e^{i\left(k_{m} x_{l}+k_{n} x_{j}\right)}}{2 i k_{n}}, \\
\mathbf{s}_{2 n, 1 m}=\delta_{n m}+\sum_{j, l=1}^{N} \beta_{j l}^{n m m} \frac{e^{i\left(k_{m} x_{l}-k_{n} x_{j}\right)}}{2 i k_{n}}, \\
\frac{\delta \mathbf{s}_{1 n, 1 m}}{\delta U(\mathbf{r})}=\sum_{l=1} \phi_{l}(y)\left(A_{l n m}+\sum_{i, j=1}^{N} B_{l n m}^{i j} e^{+i k_{n} x_{i}}\right) \psi_{m}^{(N)}(\mathbf{r}), \\
\frac{\delta \mathbf{s}_{2 n, 1 m}}{\delta U(\mathbf{r})}=\sum_{l=1} \phi_{l}(y)\left(A_{l n m}+\sum_{i, j=1}^{N} B_{l n m}^{i j} e^{-i k_{n} x_{i}}\right) \psi_{m}^{(N)}(\mathbf{r}) .
\end{gathered}
$$

In these expressions,

$$
\begin{gathered}
\beta_{i j}^{n m l} \equiv \sqrt{\frac{k_{n}}{k_{m}}} \phi_{n}\left(y_{i}\right) \phi_{m}\left(y_{j}\right) \gamma M_{i j} ; \\
A_{l n m} \equiv \delta_{l n} / \sqrt{\left(-i k_{l} x\right) /\left(2 i k_{l}\right)} ; \\
B_{l n m}^{i j} \equiv \beta_{i j}^{n m l} \exp \left(i k_{l}\left|x_{j}-x\right|\right) /\left(2 i k_{n} 2 i k_{l}\right) ;
\end{gathered}
$$

$\psi_{m}^{(N)}(\mathbf{r})$ is the $N$-impurity scattering wave function [Eq. (8)] with an electron incoming from lead 1 in mode $m$.

With the above analytical results we can proceed to plot the emittance $E_{\alpha \beta}$ and its fluctuations for impurity scattering. For comparison we have also calculated the dc conductance fluctuations from the scattering matrix derived above. For a given impurity configuration $\left\{\mathbf{r}_{i}\right\}$ where $i=1,2, \ldots, N$, generated randomly and distributed uniformly, we evaluate the quantity $M_{i j}$ by direct matrix inversion. The rest of the expressions are calculated once $M_{i j}$ is known. The transverse modes $\phi_{n}(y)$ are the usual sine functions, and we have fixed the incoming electron energy so that there are 18 propagating subbands in the quantum wire whose contributions are summed. The sample-to-sample statistical analysis is carried out by averaging many independent impurity configurations for each given $N$.

Figure 1 plots the typical sample-to-sample emittance fluctuations for $N=300$ and $\gamma=50$. This is to be compared with the usual $\mathrm{dc}$ conductance fluctuations (inset) which have an amplitude $\sim e^{2} / h$, i.e., the UCF situation. For this degree of disorder it is apparent that $E_{11}$ fluctuates between 


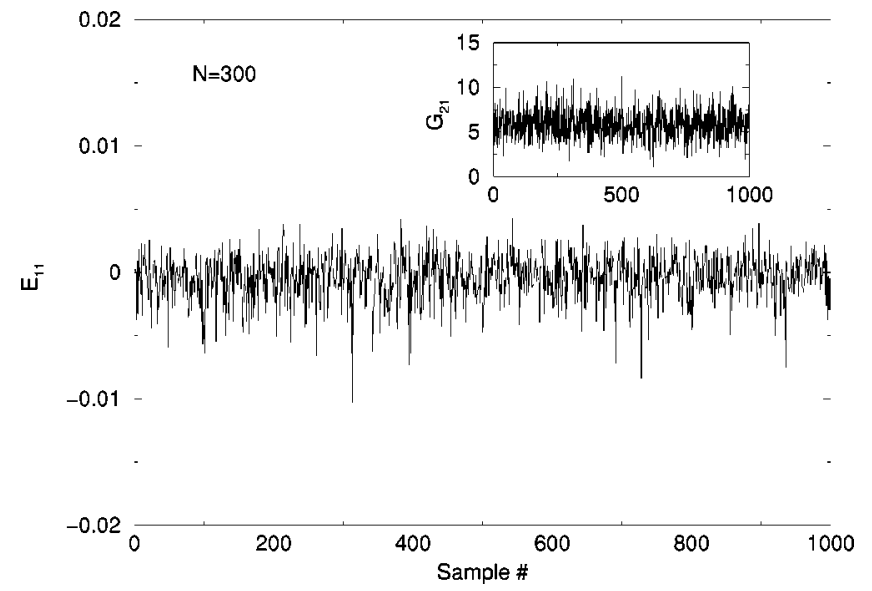

FIG. 1. Typical sample-to-sample fluctuations of the emittance $E_{11}$ with fixed the impurity number $N=300$ and strength $\gamma=50$. Inset: sample-to-sample fluctuations of the dc conductance giving rise to UCF's.

negative and positive values, indicating that the dynamic response of the disordered conductor depends on the impurity configuration. The implication is that for disordered mesoscopic conductors the ac current can be leading or lagging the ac voltage quite randomly from sample to sample.

The amplitude of the fluctuations is analyzed as $(\Delta A)^{2}$ $=\left\langle A^{2}\right\rangle-\langle A\rangle^{2}$, where $A$ is $G_{21}$ and $E_{11}$. The statistical average is over energy as well as independent impurity configurations. In Fig. 2 we plot the fluctuation amplitudes as a function of the impurity strength $\gamma$ for several different impurity numbers $N .500$ impurity configurations were averaged for each $\gamma$. Figure 2(a) clearly shows that when $\gamma$ is large enough $\Delta G_{21}$ is essentially independent of $\gamma$. For $N$ $>75$ and up to $N=500$, which we have studied, $\Delta G_{21}$ $\approx 0.8 e^{2} / h$ with less than $10 \%$ difference for different $N$ 's [see Fig. 2(a)], which is consistent with the expected UCF value for $2 \mathrm{D}$ systems $\left(0.86 e^{2} / h\right) .^{2}$ In this regime, Fig. 2(b) indicates that $\Delta E_{11}$ is also independent of $\gamma$, showing a degree of universal behavior for the dynamic response with respect to the impurity scattering strength. However, because $E_{11}$ depends on the electrostatic potential buildup inside the conductor $^{3,4}$ as reflected by the functional derivative in the LPDOS (4), $\Delta E_{11}$ is expected to be a sensitive function of the degree of disorder provided by the impurity number $N$. Our results confirm this picture as shown in Fig. 2(b).

An important quantity is the distribution function $P\left(E_{11}\right)$. Of the two contributions to $E_{11}$, namely, the external charge injection due to the time dependent disturbance and the internal response due to Coulomb interactions, the external contribution is given by the global partial density of states which is related the electron dwell time of the scattering region. ${ }^{17}$ The distribution function of the dwell time has been analyzed within the random matrix theory ${ }^{20,8}$ for chaotic cavities and within the invariant embedding formalism for 1D disordered systems. ${ }^{21}$ Its universal properties have been well studied. ${ }^{22,23}$ However, the statistical property of the internal response is much more complicated and it has been included within the constant capacitance charging model only for chaotic cavities. ${ }^{8,6}$ Here we numerically investigate
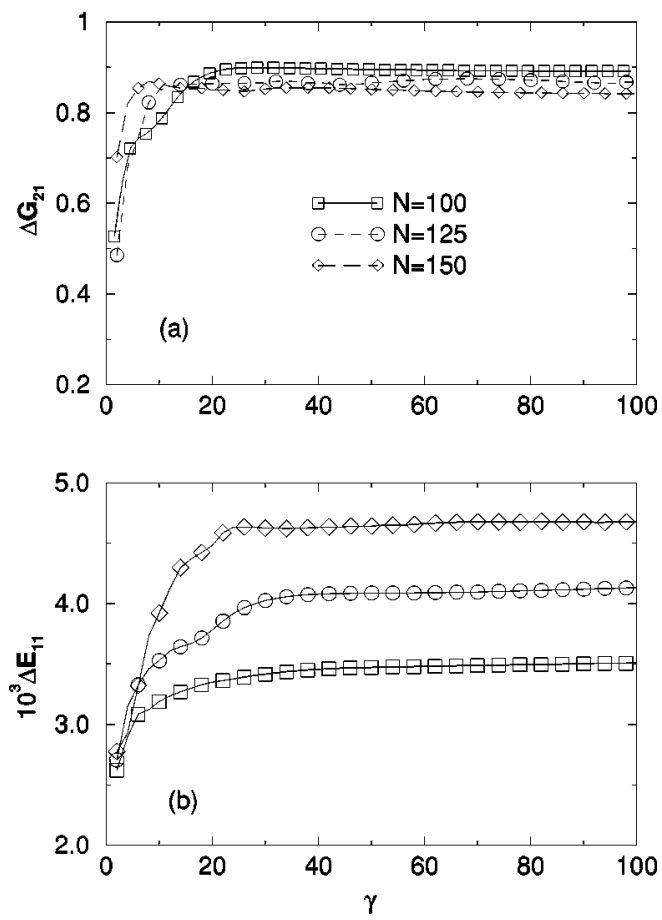

FIG. 2. For both (a) and (b), squares, $N=100$ impurities; circles, $N=125$; diamonds, $N=150$. (a) dc conductance fluctuations as a function of the impurity scattering strength $\gamma$. A clear signature of UCF's is observed with $\Delta G \approx 0.86 e^{2} / h$ without dependence on $\gamma$ and weak dependence on $N$. (b) Emittance fluctuations $\Delta E_{11}$ as a function of $\gamma$ : for large enough $\gamma, \Delta E_{11}$ is essentially independent of $\gamma$, but it strongly depends on the impurity density.

$P\left(E_{11}\right)$ from first principles, where the internal response is calculated through the LPDOS as discussed above. Figure 3 shows $P\left(E_{11}\right)$ obtained from our numerical analysis for different $N$ values. Over 9000 independent impurity configurations were averaged for each $N$. We focus on the regime where the dc transport shows UCF's. When $N$ is small, e.g., $N=100, P\left(E_{11}\right)$ centers at negative $E_{11}$ indicating a predominantly inductive dynamic response [see Fig. 3(a)]. This is consistent with the fact that the system is rather conductive for this degree of disorder. The distribution function is quite symmetric. As $N$ is increased to 300 , the larger degree of disorder makes the system less conductive, and the distribution is shifted to center near zero [Fig. 3(b)]. While the distribution is still quite symmetric, it is now wider, indicating a larger fluctuation amplitude $\Delta E_{11}$, as was seen in Fig. 2(b). When $N$ is increased further, $P\left(E_{11}\right)$ not only shifts to the center at positive values of $E_{11}$, it becomes asymmetric [Figs. 3(c) and 3(d)]. For large $N$ the disordered system is less conductive; therefore we expect a capacitive dynamic response (e.g., a parallel plate capacitor has zero dc conductance). This is clearly shown by the shift of the distribution toward positive values of emittance. In this regard, we note that the random matrix theory prediction of capacitance distribution for a one-probe chaotic cavity is also asymmetric. ${ }^{8}$ Although it seems to be quite difficult to analytically derive an expression for $P\left(E_{11}\right)$, its behavior can be understood by considering the $M_{e f f} \sim M(l / L)$ conductive channels of the disordered sample. It is reasonable to assume that these conductive channels contribute largely to the inductivelike re- 

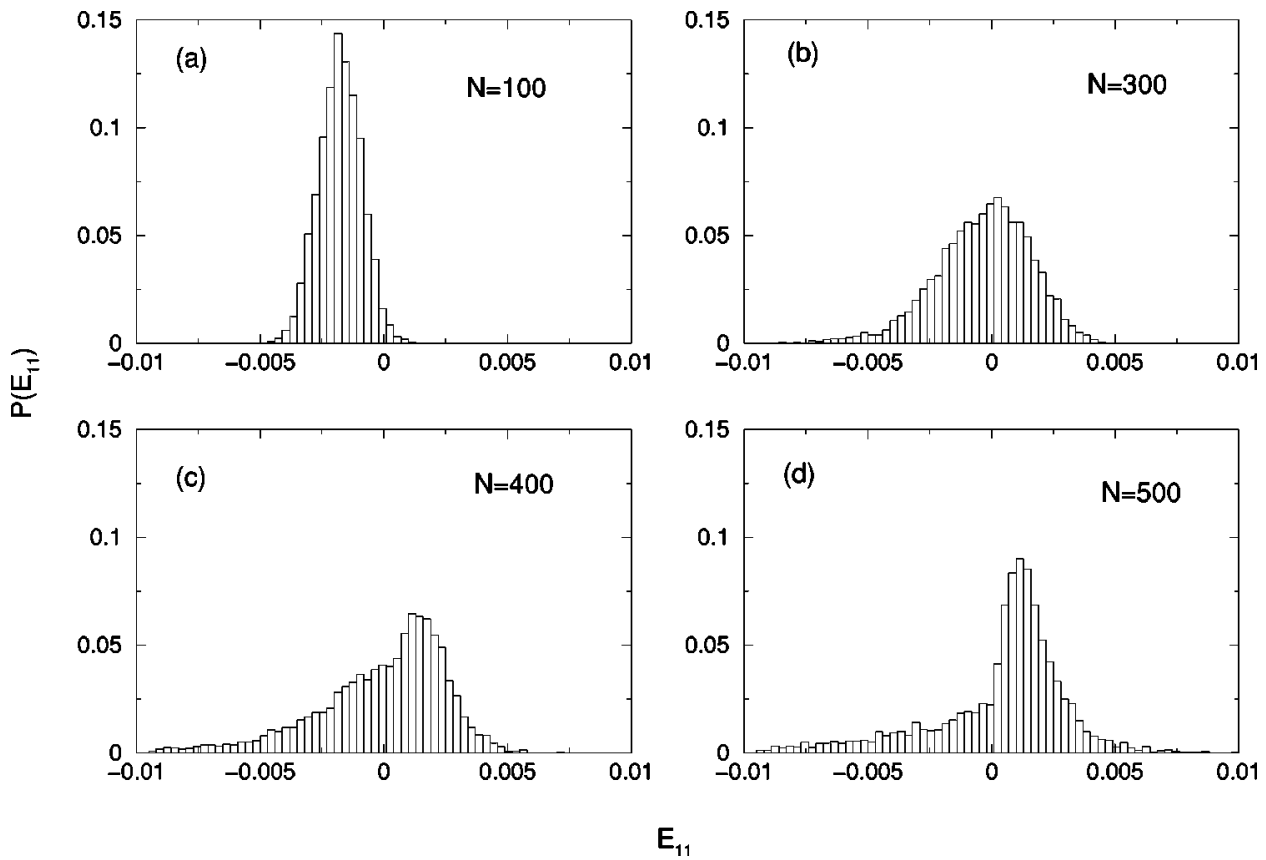

FIG. 3. The distribution function $P\left(E_{11}\right)$. For (a) $N=100$; (b) $N=300 ;$ (c) $N=400$; and (d) $N$ $=500$. As $N$ increases the distribution crosses over from a symmetric form to asymmetric, as the dynamic response changes from inductive to capacitive. Parameter $\gamma=100$ is used.

sponse. Hence, when $M_{e f f}$ is large [e.g., Fig. 3(a)] due to a smaller degree of disorder, $E_{11}$ fluctuates around its mean (which is a negative number) from sample to sample, but each sample is inductive and hence $P\left(E_{11}\right)$ is expected to be symmetric. On the other hand, when disorder increases and the distribution shifts to the center at a positive mean of $E_{11}$ [e.g., Figs. 3(c), 3(d)], there are always samples with substantial $M_{\text {eff }}$ (as long as transport stays in the mesoscopic regime) due to quantum interference and statistical distribution of the impurities, and these samples will be inductivelike in their dynamic response. Hence we expect $P\left(E_{11}\right)$ to tail into the negative $E_{11}$ region due to these samples. Therefore $P\left(E_{11}\right)$ is asymmetric for a large degree of disorder, as Fig. 3(d) shows.

In summary, we have analyzed the sample-to-sample fluctuations of emittance for two-dimensional disordered mesoscopic conductors. Our analysis was from first principles, where both external injection and internal response were computed from the space dependent partial density of states, and we have developed a Green's function technique to de- termine these quantities for impurity scattering. In the UCF regime the emittance fluctuations show some degree of generic behavior in that the fluctuation amplitude is insensitive to the scattering strength. It does depend, however, on the degree of disorder through the impurity density, which is very different from the familiar UCF in the mesoscopic transport regime. The distribution function of the emittance has been found to be quite distinct depending on the dynamic response of the conductor: for an inductivelike response the distribution is symmetric and centered in the negative region of emittance; for a capacitive response it is asymmetric and centered at a positive emittance.

We thank Professor Markus Büttiker for pointing out the contribution of the weak localization effect to the average value of the emittance. We gratefully acknowledge financial support from NSERC of Canada and FCAR of Quebec (H.G.); and RGC Grant No. HKU 7215/99P from the Hong Kong SAR (J.W.).
${ }^{1}$ S. Washburn and R.A. Webb, Adv. Phys. 35, 375 (1986).

${ }^{2}$ See, for example, Mesoscopic Phenomena in Solids, edited by B.L. Altshuler, P.A. Lee, and R.A. Webb (North-Holland, Amsterdam, 1991); C.W.J. Bennakker and H. van Houten, in Solid State Physics, Vol. 44, edited by H. Ehrenreich and D. Turnbull (Academic Press, New York, 1991), p.1; Y. Imry, in Directions in Condensed Matter Physics, edited by G. Grinstein and G. Mazenko (World Scientific, Singapore, 1986), p.101; C.W.J. Beenakker, Rev. Mod. Phys. 69, 731 (1997).

${ }^{3}$ M. Büttiker, A. Prêtre, and H. Thomas, Phys. Rev. Lett. 70, 4114 (1993); ibid. 71, 465 (1993); M. Büttiker, H. Thomas, and A. Prêtre, Z. Phys. B: Condens. Matter 94, 133 (1994).

${ }^{4}$ B.G. Wang, Jian Wang, and Hong Guo, Phys. Rev. Lett. 82, 398 (1999).
${ }^{5}$ John B. Pieper and John C. Price, Phys. Rev. Lett. 72, 3586 (1994).

${ }^{6}$ P.W. Brouwer and M. Büttiker, Europhys. Lett. 37, 441 (1997).

${ }^{7}$ Y.V. Fyodorov and H.J. Sommers, J. Math. Phys. 38, 1918 (1997).

${ }^{8}$ V.A. Gopar, P.A. Mello, and M. Büttiker, Phys. Rev. Lett. 77, 3005 (1996).

${ }^{9}$ J. Cohen and Y. Avishai, J. Phys.: Condens. Matter 7, L121 (1995).

${ }^{10}$ D.Z. Liu, B.Yu-K. Hu, C.A. Stafford, and S. Das Sarma, Phys. Rev. B 50, 5779 (1994).

${ }^{11}$ For a review, see, for example, M. Büttiker and T. Christen, in Quantum Transport in Semiconductor Submicron Structures, 
Vol. 326 of NATO Advanced Study Institute, edited by B. Kramer (Kluwer, Dordrecht, 1996).

${ }^{12}$ M. Büttiker and T. Christen, in Theory of Transport Properties of Semiconductor Nanostructures, edited by E. Schöll (Chapman and Hall, London, 1998), pp. 215-248.

${ }^{13}$ Y. Imry, Europhys. Lett. 1, 249 (1986).

${ }^{14}$ Jian Wang, Qingrong Zheng, and Hong Guo, Phys. Rev. B 55, 9770 (1997).

${ }^{15}$ Another example of an impurity in a quantum wire was given by Christen and Büttiker who treated the quantum point contact in an external ac field. See T. Christen and M. Büttiker, Phys. Rev. Lett. 77, 143 (1996).

${ }^{16}$ M. Büttiker, J. Phys.: Condens. Matter 5, 9361 (1993).
${ }^{17}$ V. Gasparian, T. Christen, and M. Büttiker, Phys. Rev. A 54, 4022 (1996).

${ }^{18} \mathrm{~S}$. Datta, Electronic Transport in Mesoscopic Systems (Cambridge University Press, New York, 1995).

${ }^{19}$ The details of the mathematical development and other results will be presented elsewhere. Tiago Dejesus, Hong Guo, and Jian Wang (unpublished).

${ }^{20}$ Y.V. Fyodorov and H.-J. Sommers, Phys. Rev. Lett. 76, 4709 (1996).

${ }^{21}$ A.M. Jayannavar, G.Y. Vijayagovindan, and N. Kumar, Z. Phys. B: Condens. Matter 75, 77 (1989).

${ }^{22}$ C. Texier and A. Comtet, Phys. Rev. Lett. 82, 4220 (1999); A. Comtet and C. Texier, J. Phys. A 30, 8017 (1997).

${ }^{23}$ Y.V. Fyodorov, Phys. Rev. B 61, 2444 (2000). 